Boise State University

ScholarWorks

$11-1-2010$

\title{
The Role of Caspases in Alzheimer's Disease; Potential Novel Therapeutic Opportunities
}

Troy T. Rohn

Boise State University 


\title{
The Role of Caspases in Alzheimer's Disease; Potential Novel Therapeutic Opportunities
}

Troy T. Rohn, Ph.D.

Boise State University

\begin{abstract}
Although apoptosis plays a critical role in molding the CNS into its final appearance and function, inappropriate activation of this pathway in the aging brain may contribute to neurodegeneration. In Alzheimer's disease (AD), an overwhelming body of evidence supports the activation of apoptosis in general, and caspases specifically as an early event that may not only contribute to neurodegeneration but also promote the underlying pathology associated with this disease. Therefore, caspase inhibitors may provide an effective strategy for treating AD. However, despite the compelling evidence indicating a role for caspases in disease progression, chronic treatment with caspase inhibitors in animal models of AD has never been undertaken. In this review the role of caspases in $\mathrm{AD}$ will be addressed, including recent studies utilizing in vivo transgenic mouse models of tauopathies. In addition, a discussion of the therapeutic value and dangers of targeting caspase inhibition in the treatment of AD using caspase inhibitors such as Q-VD-OPh will be evaluated.
\end{abstract}

Keywords: apoptosis, caspases, Alzheimer's Disease, tau, beta-amyloid, amyloid precursor protein, neurofibrillary tangles, ROS; Q-VD-OPh

\section{Overview of Alzheimer's Disease}

Recent statistics in the United States regarding Alzheimer's disease (AD) are alarming: 5.3 million people have AD with a new case diagnosed every 70 seconds and $A D$ is currently the sixth leading cause of death [1]. AD is a progressive neurodegenerative disorder characterized by confusion, disturbances in short-term memory and cognitive impairment. These early symptoms of $\mathrm{AD}$ may be overlooked because they resemble signs of natural aging. As the disease progresses, the symptoms gradually worsen until patients lose the ability to feed themselves, speak, recognize people and memory becomes almost non-existent. In addition, during the advanced stages of dementia, the impact on the lives of patients and their caregivers is enormous.

Pathologically, $\mathrm{AD}$ is a progressive neurodegenerative disorder characterized by significant losses of neurons and synapses [2]. Diagnosis is based on the extent of accumulation of senile plaques, neurofibrillary tangles (NFTs), and neuron loss [3]. NFTs consist of paired helical filaments (PHF) resulting from the hyper-phosphorylation of the microtubule-binding protein tau. As a microtubule-associated protein, tau plays an essential role in maintaining microtubule stability similar to ties along railroad tracks. However, in AD neuronal tau is aberrantly phosphorylated and proteolyzed resulting in an impairment of the normal functions of tau $[4,5]$. Besides NFTs, the other troublemakers are senile plaques composed principally of extracellular amyloid- $\beta$ (A $\beta$ ) depositions. $A \beta$ is formed following sequential cleavage of the amyloid precursor protein (APP) by two proteases termed $\beta$ - and $\gamma$-secretases. Following generation, $A \beta$ self-aggregates into insoluble $\beta$-sheet structures leading to the formation of extracellular plaques. The deposition of $\mathrm{A} \beta$ is an early event in the pathogenesis of $\mathrm{AD}$ that precedes the formation of NFTs and collectively is referred to as the "beta-amyloid hypothesis" [6]. More recently, the beta-amyloid hypothesis has been extended by suggesting that $A \beta$ - oligomers, due to their central neuropathologic role, are the major causative species for the development of $\mathrm{AD}$ [7]. The extension of the beta-amyloid hypothesis to include $\mathrm{A} \beta$ oligomers is based at least in part on the poor correlation between the number of senile plaques and cognitive decline [8]. Figure $1 \mathrm{~b}$ depicts the characteristic pathology associated with $\mathrm{AD}$, with an abundance of both plaques and tangles present in one representative brain region, the hippocampus. Interestingly, a smattering of tangles can also be observed in the age-matched non-demented control subject (arrow, Fig. 1a). The finding of NFTs in non-demented aging subjects is a well-documented finding (for example, see [9]), and suggests that the presence of a small number of NFTs in vulnerable brain regions doesn't not necessary imply neurodegeneration or disease. Although the presence 
This is an author-produced, peer-reviewed version of this article. The final publication is available at www.springerlink.com. Copyright restrictions may apply. DOI: 10.1007/s10495-010-0463-2

of both plaques and tangles is a central feature associated with $\mathrm{AD}$, the link connecting these two hallmark pathologies has remained elusive. Recent evidence suggests that caspase activation and cleavage of tau may link plaques and tangles in $\mathrm{AD}$.

\section{Caspase activation and cleavage of tau in the AD brain}

Our interest in the role of caspases in Alzheimer's began almost 10 years ago when we began investigating apoptosis as a cell death mechanism in the AD brain. At the time there was support for apoptosis as a major celldeath pathway in the $\mathrm{AD}$ brain [10], however, this was greatly disputed because the major technique to detect apoptosis, TUNEL labeling, was deemed nonspecific [11]. Therefore, we sought to examine a role for apoptosis in the AD brain utilizing caspase-cleavage site-directed antibodies. In this approach, antibodies are directed towards the active fragments of caspases or neoepitope regions of caspase substrates following cleavage. This approach was first employed by Yang el al. who, using a site-directed antibody to caspasecleaved actin, was able to demonstrate convincingly the activation of apoptotic pathways in the AD brain [12]. This was followed by a comprehensive study by Gervais et al., in which a site-directed caspase-cleavage antibody to APP demonstrated labeling in the AD brain and suggested that not only was APP a substrate for caspases but cleavage may facilitate the further production of the toxic A $\beta$ fragment [13]. Based on these studies, we applied the same approach to design a specific sitedirected antibody to fodrin, a major cytoskeletal protein expressed in neurons [14]. Application of this antibody showed widespread caspase-cleaved fodrin in the AD brain [15]. An interesting outcome from that study was the apparent correlation between caspase-cleaved fodrin and NFTs [15]. It was concluded that perhaps there was a link between caspase activation and the formation of NFTs in the AD brain. Another piece of evidence suggesting that caspases may link $\mathrm{A} \beta$ to NFTs in $\mathrm{AD}$ was the identification that tau is a major substrate for caspase cleavage, in vitro [16]. Canu et al. were able to show that there were three potential caspase cleavage sites within tau, and based on this study, we designed a caspase-cleavage site-directed antibody to the amino-terminal site within tau. Following characterization of this antibody, we were able to demonstrate immunolabeling of this antibody in the $\mathrm{AD}$ brain that was absent in age-matched nondemented control subjects [17]. This was the first demonstration of the presence of caspase-cleaved tau in AD. Following this study, we developed a hypothesis in which A $\beta$ triggers the activation of caspases leading to proteolysis of tau and the NFT formation[18].

Shortly thereafter, two studies largely confirmed our hypothesis by demonstrating that the caspase cleavage of tau is an early event in NFT evolution, and links A $\beta$ to NFT formation in the AD brain $[19,20]$. Both studies relied heavily on data obtained using identical site-directed antibodies to the $\mathrm{C}$-terminal caspase-cleavage site within tau located at amino acid residue D421. Both studies supported a general role for caspase-3 as being the major executioner protease involved in cleaving tau at this $\mathrm{C}$-terminal site. In addition to caspase- 3 , strong evidence for caspase- 6 and the cleavage of tau in the AD brain has been demonstrated [21], suggesting the cooperation of multiple members of the caspase family in the proteolysis of tau. Importantly, the use of postmortem human AD tissue in these studies makes it difficult to determine a direct relationship between the caspase-cleavage of tau and the formation of NFTs. Figure 2 summarizes the data supporting a role for caspase activation as being one link between $A \beta$ and NFT formation in the AD brain.

More recently, transgenic mice studies have provide additional evidence that caspases play a proximal role in promoting tangle formation in $\mathrm{AD}$. We utilized the $3 \mathrm{xTg}-\mathrm{AD}$ mice developed by Oddo et al. to examine more directly the role for caspases in tangle formation. 3xTg-AD mice sequentially form A $\beta$ lesions followed by tau alterations including hyperphosphorylation that closely models what occurs in the human AD brain [22, 23]. We generated $3 \mathrm{xTg}-\mathrm{AD}$ mice that overexpress the anti-apoptotic protein, $\mathrm{Bcl}-2$, specifically in neurons of the CNS and aged the mice accordingly before behavioral and pathological examination. It was determined that overexpression of Bcl-2 prevented caspase activation and cleavage of tau, limited the degree of tau hyperphosphorylation, and improved spatial memory [24]. Even more convincing then this study, are the recent studies coming out of Bradley Hyman's group using multiphoton microscopy, a technique that allows one to detect caspase activation in the living brain and follow this activation over several days. Using this powerful technique, Spires-Jones et al. were able to detect active caspases within tangle-bearing neurons in the rTg4510 mouse model of tauopathy [25]. They have been able to extend these findings by showing that even though caspases are activated with NFT-bearing neurons, these neurons do not immediately die, but exhibit significant loss of membrane integrity as indicated by the presence 
This is an author-produced, peer-reviewed version of this article. The final publication is available at www.springerlink.com. Copyright restrictions may apply. DOI: 10.1007/s10495-010-0463-2

of propidium iodide labeling [26]. Taken together, these studies employing animal models of AD provide strong experimental support for a role in caspases in the etiology associated with $\mathrm{AD}$, and thus, are potential therapeutic targets for the treatment of this disease.

\section{Caspases as Therapeutic Targets in the Treatment of AD}

The potential for caspase inhibitors as therapeutic agents is not unique to AD. Indeed, for many years caspase inhibitors such as N-benzyloxycarbonyl-Val-Ala-Asp fluoromethyl-ketone (Z-VAD) were being investigated for the treatment of a number of neurodegenerative disorders including amyotrophic lateral sclerosis (ALS) [27], Huntington's disease [28], Parkinson's disease (PD) [29], and finally acute neurologic diseases including ischemia or traumatic injury (for review, see [30]). Unfortunately, clinical development of Z-VAD was discontinued following the recognition that metabolism of Z-VAD produces liver damage following the production of the toxic compound, fluoroacetate [31].

Following the disappointment of Z-VAD, a number of other caspase inhibitors have been developed with the goal in mind of being safer and more selective. Quinolyl-valyl-Omethylaspartyl-[- 2, 6-difluorophenoxy]-methyl ketone (QVD-OPh) is a newer, third-generation broad-spectrum caspase inhibitor that has potential as a therapeutic compound [32]. Q-VD-OPh appears to be far superior to Z-VAD in many respects including greater potency, selectivity, stability and cell permeability [32]. Q-VD-OPh also appears to be less toxic than Z-VAD even at very high concentrations [33]. Most importantly, Q-VD-OPh appears to be able to cross the blood-brain barrier, which is always a central issue when developing a drug for treatment of a CNS disorder. Evidence for neuroprotection included studies demonstrating efficacy of Q-VDOPh in animal models of Parkinson's, Huntington's disease and stroke $[34,35]$. Therefore, QVD-OPh would seem to be an ideal candidate to test whether pharmacological inhibition of caspases in vivo can prevent the pathology associated with $\mathrm{AD}$. The critical next step is to test directly whether Q-VD-OPh prevents neurodegeneration or other aspects of pathology using animal models of AD.

Another promising compound that is currently under evaluation is minocycline, a second-generation tetracycline. In comparison to Q-VD-OPh, minocycline is much further along as an investigational drug for the treatment of neurodegenerative disorders and in some cases human clinical trials have begun. For example, minocycline is currently being tested in a Phase II PD clinical trial as well as a Phase I/II clinical trial for spinal cord injury (for a recent review, see [36]). In addition, recently a Phase III trail involving minocycline as a therapeutic agent for the treatment of ALS was completed. Unfortunately, the results of the trial were negative as the authors found that patients on minocycline declined at a more rapid rate than the placebo group [37]. These results are disappointing and certainly will have implications for potential future clinical trials of minocycline in AD. Nonetheless, there is ample data to suggest minocycline may be a suitable treatment option for $\mathrm{AD}$. In one study by Choi et al., the authors reported that minocycline administration improved behavior deficits in $A \beta$ infused rats [38]. Minocycline also has been shown to improve behavioral deficits while reducing the level of microglial activation in a mouse of model cerebral microvascular amyloid, although there was no apparent reduction in $A \beta$ deposition [39]. In yet another study using transgenic mice harboring the human APPsw and V717F mutations, treatment with minocycline had a positive effect on reducing microglial activation but did not attenuate $\mathrm{A} \beta$ deposition or behavioral deficits in APP mice [40]. Younger mice faired better, exhibiting improved behavioral and microglial suppression following minocycline treatment [40]. Finally, a recent study showed that in primary cortical neurons minocycline prevented A $\beta$-induced death, caspase-3 activation, and reduced the levels of caspase-cleaved tau [41]. The same authors showed that in vivo treatment of transgenic mice overexpressing human tau with minocycline reduced levels of tau phosphorylation and tau cleavage by caspase-3 [41]. Taken together, these studies support a preventative effect of minocycline on caspase activation, pathological changes to tau and the inflammation associated with AD with little effect on $A \beta$ deposition. This suggests that the mechanism of action of minocycline may lie downstream to $A \beta$ production and deposition.

Mechanistically, current data suggests minocycline inhibits apoptosis by preventing the release of cytochrome c from mitochondria and subsequent caspase-3 activation [42, 43], although how minocycline is actually accomplishing this feat is presently unknown. Minocycline also exhibits strong anti-inflammatory actions particularly in preventing reactive microgliosis $[44,45]$ a key feature that has been associated with AD [46]. Therefore, minocycline would seem an ideal compound to be tested in the treatment of AD based partly on its putative mechanisms of action. Table 1 summarizes the similarities and differences between Q-VD-OPh and minocycline with regards to their potential as therapeutic agents for the treatment of AD. 
This is an author-produced, peer-reviewed version of this article. The final publication is available at www.springerlink.com. Copyright restrictions may apply. DOI: 10.1007/s10495-010-0463-2

\section{Possible toxicities associated with caspase inhibitors as treatment compounds for AD}

An obvious point of concern of utilizing caspase inhibitors such as Q-VD-OPh in the treatment of AD are potential toxicities related to the inhibition of apoptosis in other cell types. Chronic administration of any caspase inhibitor for a time period exceeding 14 days has never been reported in any animal model or in vivo disease system. Clearly, one might predict the deleterious effects of a non-selective caspase inhibitor, given the important role of caspases in overall tissue homeostasis. However, based on available evidence, Q-VD-OPh and other caspase inhibitors may have a more limited toxicity profile than predicted. First, Chauvier et al., found that after acute treatment of Q-VD$\mathrm{OPh}$ in mice, all organs were normal suggesting a lack of toxicity [33]. Second, the caspase inhibitor IDN-6556, which shares structural similarities with Q-VD-OPh, was reported to be safe and well tolerated in humans (up to 10 $\mathrm{mg} / \mathrm{kg} /$ infusion for a single dose) in a phase I clinical trial [47]. This was a 14-day study in which there were no apparent drug-related adverse events. Third, the potential for a lack of toxicity by Q-VD-OPh could be predicted based on the fact that caspase inhibitors cannot block any caspase-independent cell death pathways. Similar to IDN6556 , minocycline has a proven track record for safety in a number of clinical trials involving humans. For example, treatment with minocycline in Phase I clinical trials for ALS and PD has been successfully completed with little toxicity noted (reviewed in [36]). However, in a recent Phase III trial, patients on minocycline declined more quickly than those on placebo [37]. In addition, a Phase II trial for PD indicated a decrease tolerability of minocycline in the treatment group [48]. It should be noted that differences in toxicity between minocycline and QVD-OPh may be expected if one considers the multiplicity of putative actions of minocycline versus Q-VD-OPh, which is extremely selective for only inhibiting caspases. In sum, the potential for toxicity and untoward side effects with caspase inhibitors is an obvious concern. Until comprehensive studies with caspase inhibitors, such as Q-VD$\mathrm{OPh}$ are carried out in animal models of $\mathrm{AD}$ one can only speculate on the relative efficacy versus toxicity that may occur following chronic treatment.

\section{Conclusions}

Mounting evidence suggests the involvement of caspases in the disease process associated with AD. The activation of caspases may be responsible for the neurodegeneration associated with $\mathrm{AD}$ and several recent studies have suggested that caspases may also play a role in promoting the disease mechanisms associated with this disease. Thus, caspase activation and cleavage of critical cellular proteins associated with AD pathology may facilitate both the production of $A \beta$ as well as the formation of NFTs. Because the activation of caspases in AD may be a proximal event that is not just associated with neurodegeneration, caspases are potential therapeutic targets for the treatment of this disorder. Presently, there are only a few FDA-approved medications available for patients afflicted with AD and these medications are palliative in nature. Although there are numerous strategies under development that involve the beta-amyloid hypothesis, it is still not known whether these strategies will positively impact the disease. Given the recent phase III clinical trial failures involving the beta-amyloid hypothesis [49], a prudent approach to developing therapeutics for $\mathrm{AD}$ should be aimed at other potential targets. Over a decade of research has supported a role for the activation of caspases in the AD brain, and recent studies has indicated an involvement in this pathway in promoting the pathology underlying this disease. However, to date, studies testing the potential of anti-apoptotic compounds have yet to be realized. This may be partly due to real concerns related to potential side effects associated with the chronic treatment of apoptotic inhibitors including most importantly, the potential for tumor formation. Two compounds, minocycline and Q-VD-OPh, seem ideal candidates to test in animal models of AD. Until studies in animal models of $\mathrm{AD}$ are carried out, we will never know whether the risk/reward associated with caspase inhibitors such as Q-VD-OPh are worthy of future drug development for the treatment of $\mathrm{AD}$. With the number of Americans with Alzheimer's expected to triple by the middle of the century [1], the risk of testing apoptotic inhibitors as a treatment strategy seems well worth the effort. 
This is an author-produced, peer-reviewed version of this article. The final publication is available at www.springerlink.com. Copyright restrictions may apply. DOI: 10.1007/s10495-010-0463-2

\section{References Cited}

1. (2009) Alzheimer's disease facts and figures. Alzheimers Dement 5:234-270.

2. Duyckaerts C, Delatour B, Potier MC. (2009) Classification and basic pathology of Alzheimer disease. Acta Neuropathol 118:5-36.

3. Mirra SS, Heyman A, McKeel D, et al. (1991) The consortium to establish a registry for Alzheimer's disease (CERAD) part II. Standardization of the neuropathological assessment of

Alzheimer's disease. Neurol 41:479-486.

4. Trojanowski JQ, Schmidt ML, Shin R-W, Bramblett GT, Goedert M, Lee M-Y. (1993) From pathological marker to potential mediator of neuronal dysfunction and degeneration in Alzheimer's disease. Clinical Neuroscience 1:184191.

5. Bramblett GT, Goedert M, Jakes R, Merrick SE, Trojanowski JQ, Lee VM. (1993) Abnormal tau phosphorylation at Ser396 in Alzheimer's disease recapitulates development and contributes to reduced microtubule binding. Neuron 10:1089-1099.

6. Golde TE, Dickson D, Hutton M. (2006) Filling the gaps in the abeta cascade hypothesis of Alzheimer's disease. Curr Alzheimer Res 3:421-430.

7. Lambert MP, Barlow AK, Chromy BA, et al. (1998) Diffusible, nonfibrillar ligands derived from Abeta1-42 are potent central nervous system neurotoxins. Proc Natl Acad Sci U S A 95:6448-6453.

8. Arriagada PV, Growdon JH, Hedley-Whyte ET, Hyman BT. (1992) Neurofibrillary tangles but not senile plaques parallel duration and severity of Alzheimer's disease. Neurology 42:631-639.

9. Price JL, Morris JC. (1999) Tangles and plaques in nondemented aging and "preclinical" Alzheimer's disease. Ann Neurol 45:358-368.

10. Su JH, Anderson AJ, Cummings BJ, Cotman CW. (1994) Immunohistochemical evidence for DNA fragmentation in neurons in the AD brain. Neuroreport 5:2529-2533.

11. Charriaut-Marlangue C, Ben-Ari Y. (1995) A cautionary note on the use of the TUNEL stain to determine apoptosis. Neuroreport 7:61-64.

12. Yang F, Sun X, Beech W, et al. (1998) Antibody to caspase-cleaved actin detects apoptosis in differentiated neuroblastoma and plaque-associated neurons and microglia in Alzheimer's disease (see comments). Am J Pathol 152:379-389.

13. Gervais FG, Xu D, Robertson GS, et al. (1999) Involvement of caspases in proteolytic cleavage of Alzheimer's amyloid- beta precursor protein and amyloidogenic A beta peptide formation. Cell 97:395-406.

14. Goodman SR, Zagon IS, Coleman DB, McLaughlin PJ. (1986) Spectrin expression inneuroblastoma cells. Brain Res Bull 16:597-602.

15. Rohn TT, Head E, Su JH, et al. (2001) Correlation between caspase activation and neurofibrillary tangle formation in Alzheimer's disease. Am J Pathol 158:189-198.

16. Canu N, Dus L, Barbato C, et al. (1998) Tau cleavage and dephosphorylation in cerebellar granule neurons undergoing apoptosis. J Neurosci 18:7061-7074.

17. Rohn TT, Rissman RA, Davis MC, Kim Y-E, Cotman C, Head E. (2002) Caspase-9 Activation and caspase cleavage of tau in the Alzheimer's disease brain. Neurobiol Dis 11:341-354. 
This is an author-produced, peer-reviewed version of this article. The final publication is available at www.springerlink.com. Copyright restrictions may apply. DOI: 10.1007/s10495-010-0463-2

18. Rohn TT, Rissman RA, Head E, Cotman CW. (2002) Caspase activation in the Alzheimer's disease brain: tortuous and torturous. Drug News Perspect 15:549-557.

19. Gamblin TC, Chen F, Zambrano A, et al. (2003) Caspase cleavage of tau: linking amyloid and neurofibrillary tangles in Alzheimer's disease. Proc Natl Acad Sci U S A 100:10032-10037.

20. Rissman RA, Poon WW, Blurton-Jones M, et al. (2004) Caspase-cleavage of tau is an early event in Alzheimer disease tangle pathology. J Clin Invest 114:121-130.

21. Guo H, Albrecht S, Bourdeau M, Petzke T, Bergeron C, LeBlanc AC. (2004) Active caspase-6 and caspase-6cleaved tau in neuropil threads, neuritic plaques, and neurofibrillary tangles of Alzheimer's disease. Am J Pathol 165:523-531.

22. Oddo S, Caccamo A, Kitazawa M, Tseng BP, LaFerla FM. (2003) Amyloid deposition precedes tangle formation in a triple transgenic model of Alzheimer's disease. Neurobiol Aging 24:1063-1070.

23. Oddo S, Caccamo A, Shepherd JD, et al. (2003) Triple-transgenic model of Alzheimer's disease with plaques and tangles: intracellular Abeta and synaptic dysfunction. Neuron 39:409-

421.

24. Rohn TT, Vyas V, Hernandez-Estrada T, Nichol KE, Christie LA, Head E. (2008) Lack of pathology in a triple transgenic mouse model of Alzheimer's disease after overexpression of the anti-apoptotic protein Bcl-2. J Neurosci 28:3051-3059.

25. Spires-Jones TL, de Calignon A, Matsui T, et al. (2008) In vivo imaging reveals dissociation between caspase activation and acute neuronal death in tangle-bearing neurons. J Neurosci 28:862-867.

26. de Calignon A, Spires-Jones TL, Pitstick R, Carlson GA, Hyman BT. (2009) Tanglebearing neurons survive despite disruption of membrane integrity in a mouse model of tauopathy. J Neuropathol Exp Neurol 68:757-761.

27. Li M, Ona VO, Guegan C, et al. (2000) Functional role of caspase-1 and caspase-3 in an ALS transgenic mouse model (see comments). Science 288:335-339.

28. Ona VO, Li M, Vonsattel JP, et al. (1999) Inhibition of caspase-1 slows disease progression in a mouse model of Huntington's disease. Nature 399:263-267.

29. Schierle GS, Hansson O, Leist M, Nicotera P, Widner H, Brundin P. (1999) Caspase inhibition reduces apoptosis and increases survival of nigral transplants. Nat Med 5:97-100.

30. Friedlander RM. (2003) Apoptosis and caspases in neurodegenerative diseases. N Engl J Med 348:1365-1375.

31. Van Noorden CJ. (2001) The history of Z-VAD-FMK, a tool for understanding the significance of caspase inhibition. Acta Histochem 103:241-251.

32. Caserta TM, Smith AN, Gultice AD, Reedy MA, Brown TL. (2003) Q-VD-OPh, a broad spectrum caspase inhibitor with potent antiapoptotic properties. Apoptosis 8:345-352.

33. Chauvier D, Ankri S, Charriaut-Marlangue C, Casimir R, Jacotot E. (2007) Broad-spectrum caspase inhibitors: from myth to reality? Cell Death Differ 14:387-391.

34. Yang L, Sugama S, Mischak RP, et al. (2004) A novel systemically active caspase inhibitor attenuates the toxicities of MPTP, malonate, and 3NP in vivo. Neurobiol Dis 17:250-259.

35. Braun JS, Prass K, Dirnagl U, Meisel A, Meisel C. (2007) Protection from brain damage and bacterial infection in murine stroke by the novel caspase-inhibitor Q-VD-OPH. Exp Neurol 206:183-191.

36. Kim HS, Suh YH. (2009) Minocycline and neurodegenerative diseases. Behav Brain Res 196:168-179. 
This is an author-produced, peer-reviewed version of this article. The final publication is available at www.springerlink.com. Copyright restrictions may apply. DOI: 10.1007/s10495-010-0463-2

37. Gordon PH, Moore DH, Miller RG, et al. (2007) Efficacy of minocycline in patients with amyotrophic lateral sclerosis: a phase III randomised trial. Lancet Neurol 6:1045-1053.

38. Choi Y, Kim HS, Shin KY, et al. (2007) Minocycline Attenuates Neuronal Cell Death and Improves Cognitive Impairment in Alzheimer's Disease Models. Neuropsychopharmacology.

39. Fan R, Xu F, Previti ML, et al. (2007) Minocycline reduces microglial activation and improves behavioral deficits in a transgenic model of cerebral microvascular amyloid. J Neurosci 27:3057-3063.

40. Seabrook TJ, Jiang L, Maier M, Lemere CA. (2006) Minocycline affects microglia activation, Abeta deposition, and behavior in APP-tg mice. Glia 53:776-782.

41. Noble W, Garwood C, Stephenson J, Kinsey AM, Hanger DP, Anderton BH. (2009) Minocycline reduces the development of abnormal tau species in models of Alzheimer's disease. Faseb J 23:739-750.

42. Zhu S, Stavrovskaya IG, Drozda M, et al. (2002) Minocycline inhibits cytochrome c release and delays progression of amyotrophic lateral sclerosis in mice. Nature 417:74-78.

43. Teng YD, Choi H, Onario RC, et al. (2004) Minocycline inhibits contusion-triggered mitochondrial cytochrome c release and mitigates functional deficits after spinal cord injury. Proc Natl Acad Sci U S A 101:3071-3076.

44. Tikka TM, Koistinaho JE. (2001) Minocycline provides neuroprotection against Nmethyl- D-aspartate neurotoxicity by inhibiting microglia. J Immunol 166:7527-7533.

45. Tikka T, Fiebich BL, Goldsteins G, Keinanen R, Koistinaho J. (2001) Minocycline, a tetracycline derivative, is neuroprotective against excitotoxicity by inhibiting activation and proliferation of microglia. J Neurosci 21:25802588 .

46. Streit WJ. (2005) Microglia and neuroprotection: implications for Alzheimer's disease. Brain Res Brain Res Rev 48:234-239.

47. Pockros PJ, Schiff ER, Shiffman ML, et al. (2007) Oral IDN-6556, an antiapoptotic caspase inhibitor, may lower aminotransferase activity in patients with chronic hepatitis C. Hepatology 46:324-329.

48. Investigators NN-P. (2008) A pilot clinical trial of creatine and minocycline in early Parkinson disease: 18month results. Clin Neuropharmacol 31:141-150.

49. Frisoni GB, Delacourte A. (2009) Neuroimaging outcomes in clinical trials in Alzheimer's disease. J Nutr Health Aging 13:209-212.

50. Butterfield DA. (2002) Amyloid beta-peptide (1-42)-induced oxidative stress and neurotoxicity: implications for neurodegeneration in Alzheimer's disease brain. A review. Free Radic Res 36:1307-1313.

51. Smith DG, Cappai R, Barnham KJ. (2007) The redox chemistry of the Alzheimer's disease amyloid beta peptide. Biochim Biophys Acta 1768:1976-1990. 
This is an author-produced, peer-reviewed version of this article. The final publication is available at www.springerlink.com. Copyright restrictions may apply. DOI: 10.1007/s10495-010-0463-2

Table 1. Comparisons of Q-VD-OPh and minocycline as potential compounds for the treatment of Alzheimer's disease

\begin{tabular}{|l|l|l|l|}
\hline Compound & \multicolumn{1}{|c|}{$\begin{array}{c}\text { Mechanism of } \\
\text { Action }\end{array}$} & \multicolumn{1}{c|}{ Advantages } & \multicolumn{1}{c|}{ Disadvantages } \\
\hline Q-VD-OPh & $\begin{array}{l}\text { Broad pan-caspase inhibitor } \\
\text { with no cross-reactivity to } \\
\text { other proteases including } \\
\text { cathepsins, calpains, or } \\
\text { granzyme B. }\end{array}$ & $\begin{array}{l}\text { Proven caspase inhibitor with } \\
\text { very select mechanism of } \\
\text { action. Great improvements } \\
\text { over older compounds such as } \\
\text { Z-VAD including potency, } \\
\text { toxicity, and stability. }\end{array}$ & $\begin{array}{l}\text { Transport into the CNS, } \\
\text { bioavailability, and } \\
\text { selectivity for specific } \\
\text { caspases could be } \\
\text { problematic. Selection } \\
\text { between "good" versus } \\
\text { "bad" apoptosis. Cost } \\
\text { prohibitive for long-term } \\
\text { study (3-24 months). Little } \\
\text { in vivo data available } \\
\text { compared to minocycline. }\end{array}$ \\
\hline Minocycline & $\begin{array}{l}\text { 1) Anti-apoptotic actions; } \\
\text { both caspase-dependent and } \\
\text { independent } \\
\text { 2) Anti-inflammatory } \\
\text { actions; suppression of } \\
\text { microglia and inhibition of T } \\
\text { lymphocytes } \\
\text { 3) Inhibition of p38 MAPK } \\
\text { activation in microglia }\end{array}$ & $\begin{array}{l}\text { Orally available and } \\
\text { demonstrated effectiveness in a } \\
\text { number of other model } \\
\text { degenerative diseases. Proven } \\
\text { tract record for safety in } \\
\text { humans. Potential dual } \\
\text { functions as apoptotic inhibitor } \\
\text { and anti-inflammatory agent } \\
\text { may beneficial in treating AD. }\end{array}$ & $\begin{array}{l}\text { Multiplicity of actions } \\
\text { may lead to untoward } \\
\text { side effects. Recent ALS } \\
\text { Phase III clinical trial with } \\
\text { minocycline was negative. } \\
\text { Clinical trials in PD and } \\
\text { Huntington's disease have } \\
\text { revealed potential safety } \\
\text { concerns in humans. }\end{array}$ \\
\hline
\end{tabular}


Figure 1, Rohn, TT

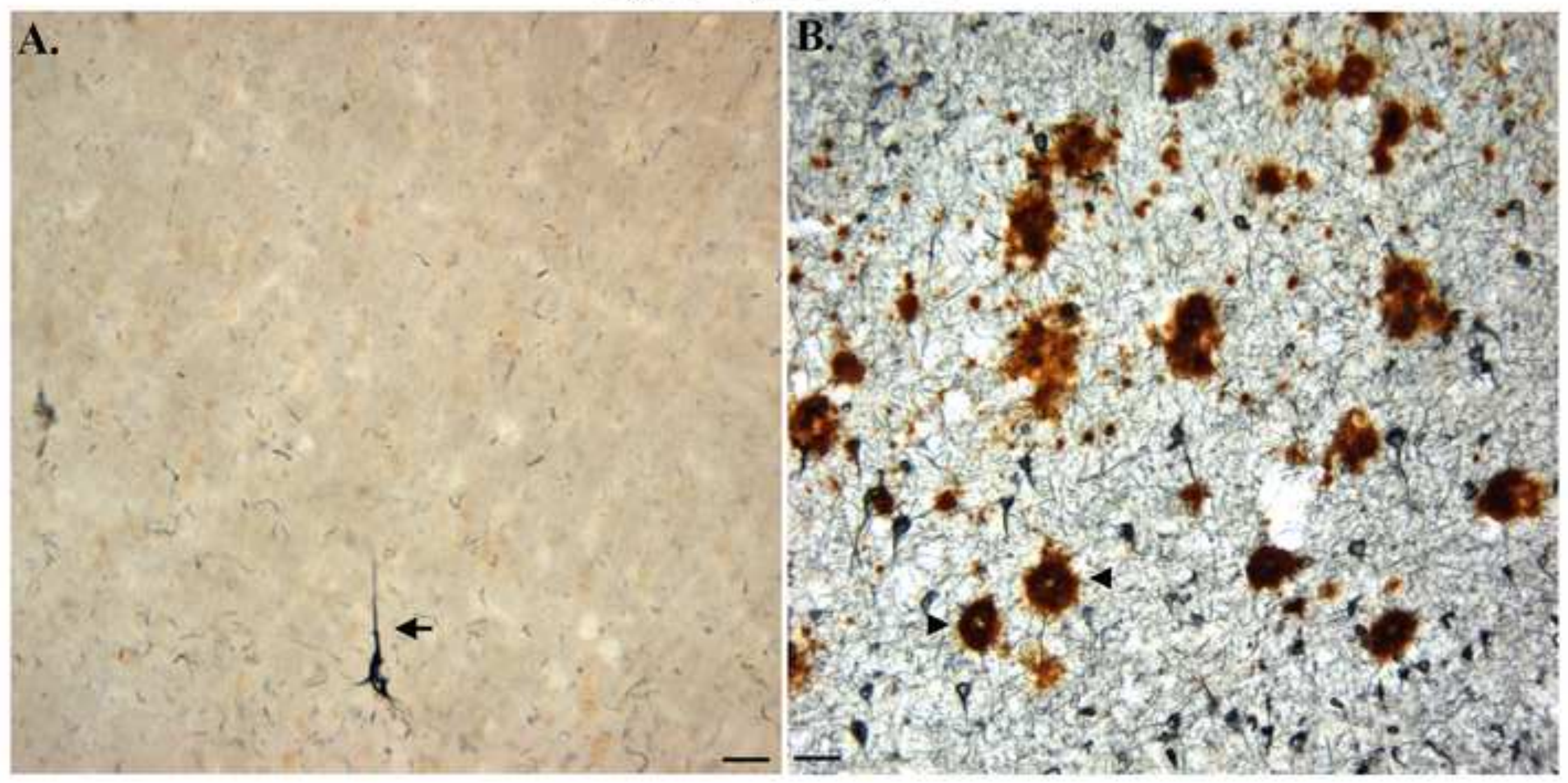




\section{Figure 2, Rohn TT}

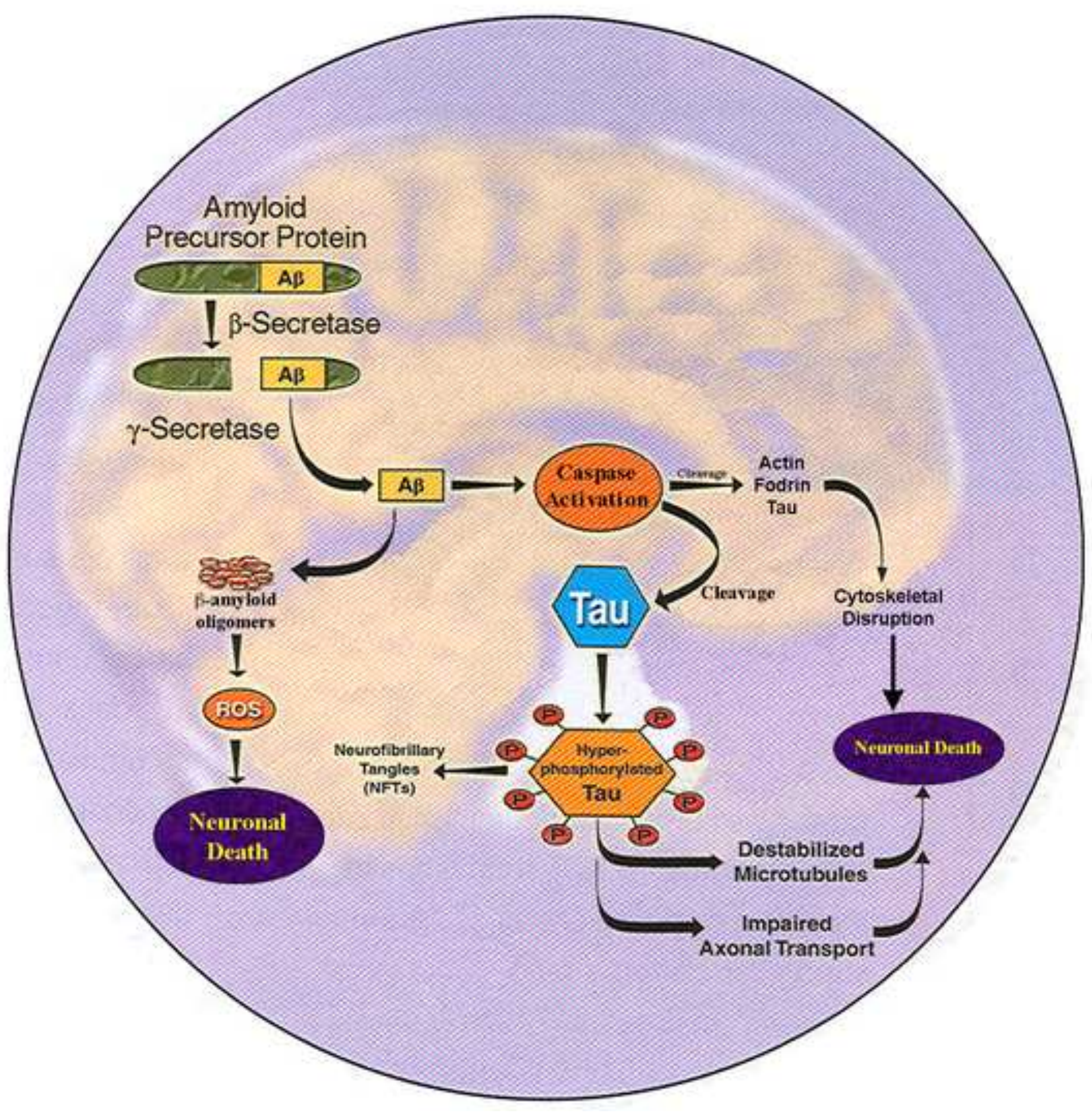


This is an author-produced, peer-reviewed version of this article. The final publication is available at www.springerlink.com. Copyright restrictions may apply. DOI: 10.1007/s10495-010-0463-2

\section{Figure Legends}

Fig. 1 Senile plaques and neurofibrillary tangles are the hallmarks of Alzheimer's disease

Representative staining from an age-matched control subject (a) or an individual with severe AD (b). In AD, widespread labeling of senile plaques composed principally of the $A \beta$ peptide (brown staining) along with numerous NFTs composed of truncated and hyperphosphorylated tau (blue staining) can be seen in the entorhinal cortex. Often, one can observe a few NFTs along with neuropil threads in the absence of A $\beta$ plaques in age-matched controls. However, the difference clearly being the number of NFTs observed as compared to the AD brain.

Fig. 2 Putative pathway of NFT formation and neuronal cell death in Alzheimer's disease.

According to the $A \beta$ hypothesis, $A \beta$ synthesis following cleavage by $\beta$ and $\beta$ secretases of the APP molecule leads to all subsequent downstream events. In one scenario, $A \beta$ aggregates into oligomers leading to the production of reactive oxygen species (ROS), oxidative stress and eventually cell death [50,51]. Alternatively or simultaneously, $\mathrm{A} \beta$ may lead to caspase activation through the initiation of apoptosis. The activation of caspases leads to the cleavage of critical cellular proteins including actin, fodrin and most importantly tau. The cleavage of these proteins may result in the breakdown of the cytoskeleton while the cleavage of tau may facilitate its hyperphosphorylation, leading to further instability in the cytoskeleton. Disruption of the microtubule network may also lead to impaired axonal transport and eventually neuronal cell death. Not shown is the fact that caspases have also been shown to cleave APP and thus, facilitate the further production of A $\beta$ peptide [13]. 